\title{
NEW APPROACH TO UNIFORMLY QUASI CIRCULAR MOTION OF QUASI VELOCITY BIHARMONIC MAGNETIC PARTICLES IN THE HEISENBERG SPACE
}

\author{
Talat Körpinar ${ }^{1}$ \\ ${ }^{1}$ Mus Alparslan Universitesi
}

July 26, 2020

\begin{abstract}
We firstly discuss the unchanged quasi direction motion (UDQM) with biharmonicity condition in the Heisenberg space. We define the energy of velocity magnetic particles and some Lorentz fields. Also, we construct the new relationship between the Fermi-Walker parallel transportation and the unchanged quasi direction motion in the Heisenberg space. In other words, we obtain the applied geometric characterization for the unchanged quasi direction motion of biharmonic velocity magnetic particles in the Heisenberg space. This concept also boosts to discover some physical and geometrical characterizations belonging to the particle such as the magnetic motion, the electrical energy functional, the torque, and the Poynting vector. Finally, we obtain electrical energy with respect to its electric field and energy flux density in the radial direction.
\end{abstract}

\section{Hosted file}

Paper.pdf available at https://authorea.com/users/295597/articles/472322-new-approach-touniformly-quasi-circular-motion-of-quasi-velocity-biharmonic-magnetic-particles-in-theheisenberg-space 
figures/QCFOSI00/QCFOSI00-eps-converted-to.pdf 
figures/QB8GH503/QB8GH503-eps-converted-to.pdf 
figures/QB8GEK02/QB8GEK02-eps-converted-to.pdf 
figures/QB8GDX01/QB8GDX01-eps-converted-to.pdf 
figures/QB8G9600/QB8G9600-eps-converted-to.pdf 